\title{
Verhaltenskodex zur medizinischen und unternehmerischen Verantwortung der Ärzte
}

Alphons Beat Schnyder ${ }^{a}$, Ulrich Schnyder ${ }^{b}$

a Dr. rer. pol., Geschäftsführer von Meta-Cultura Zürich

b Prof. Dr. med., Klinikdirektor der Klinik für Psychiatrie und Psychotherapie, UniversitätsSpital Zürich
Korrespondenz:

Dr. Alphons Beat Schnyder Meta-Cultura

Quellenstrasse 27

CH-8005 Zürich

Tel. 0442733366

schnyder@meta-cultura.ch
«Ich schwöre und rufe Apollon, den Arzt, und Asklepios und Hygieia und Panakeia und alle Götter und Göttinnen zu Zeugen an, dass ich diesen Eid und diesen Vertrag nach meiner Fähigkeit und nach meiner Einsicht erfüllen werde» ist der erste Satz im Eid des Hippokrates. Der Eid ist das erste sittliche Grundgesetz des Arztberufes und wurde nach dem griechischen Arzt Hippokrates von Kos benannt.

Die heutigen Ärzte schwören nicht mehr auf die griechischen Götter und Göttinnen. Einige haben inzwischen den Ruf erworben, sich selber als «Götter in Weiss» zu verstehen. Für andere stehen die monetären Aspekte der Medizin im Vordergrund und sie verfolgen entsprechend primär kommerzielle Interessen. Für die grosse Mehrheit der Ärzte sind jedoch ethische Fragen in Bezug auf ihre Berufsausübung ein zentrales Thema - und zwar nicht erst, seitdem sich marktwirtschaftlich orientierte Unternehmen als Folge der Finanz- und Wirtschaftskrise wieder vermehrt auf ethische Werte zurückbesinnen.

Folgende Entwicklungen werden die Bedeutung der Ethik in der Ärzteschaft in den nächsten Jahren deutlich verstärken: Im schweizerischen Gesundheitswesen sind verschiedene Reformen in Gang, welche die Rahmenbedingungen für die ärztliche Tätigkeit stark verändern werden. Die Einführung von schweizweit gültigen diagnosespezifischen Fallpauschalen bringt mehr Leistungs- und Konkurrenzdruck in die Spitäler. In der ambulanten Versorgung wird der unternehmerische Handlungsspielraum zunehmend reguliert. Insgesamt dürfte sich der Wettbewerb unter den verschiedenen Leistungsanbietern entsprechend verschärfen. Es drohen Einbussen in Bezug auf die Qualität der Patientenversorgung. Der Trend zur Ökonomisierung und Kommerzialisierung der Medizin dürfte sich ausweiten. Und generell wird der Kampf um die vorhandenen Mittel sehr wahrscheinlich deutlich zunehmen.

Die hohe Innovationskraft der Pharmaindustrie und der medizinisch-technischen Industrie wird anhalten. Die wissenschaftliche Forschung und Entwicklung wird die Medizin laufend mit neuem Wissen versorgen. Damit sind die praktizierenden Ärzte weiterhin herausgefordert, $u$.a. die im schweizerischen Krankenversicherungsgesetz verankerte Zielsetzung einer wirksamen, zweckmässigen und wirtschaftlichen medizinischen Versorgung im Rahmen der zur Verfügung stehenden Mittel sicherzustellen.

Angesichts dieser Entwicklungen wird eine klare und transparente Werteorientierung wichtiger denn je, nämlich einerseits eine verantwortungsvolle ärzt- liche Haltung in der medizinischen Versorgung, Lehre und Forschung sowie andererseits eine unternehmerische Ethik der Ärzte. Aus diesem Grund hat der Zürcher Kreis für fortschrittliches Spitalmanagement einen Verhaltenskodex zur medizinischen und unternehmerischen Verantwortung der Ärzte formuliert. Die Unterzeichnenden dieses Verhaltenskodexes machen es sich zur Aufgabe, diese ethischen Leitsätze in ihrem Tätigkeitsfeld vorbildlich zu leben. Zudem beabsichtigen sie, in der Ärzteschaft die ethische Reflexion und Diskussion anzuregen und die Kolleginnen und Kollegen für die Anwendung des Verhaltenskodexes zu motivieren.

Unterzeichner des Verhaltenskodexes des Zürcher Kreises für fortschrittliches Spitalmanagement

Prof. Dr. Ulrich Schnyder, Klinikdirektor, UniversitätsSpital Zürich*

Dr. Christoph Nöthiger, Leitender Arzt, UniversitätsSpital Zürich *

Dr. Peter Steiger, Leitender Arzt, UniversitätsSpital Zürich *

Dr. Andreas Andreae, Ärztlicher Direktor, Integrierte Psychiatrie Winterthur

Dr. Angelo Barrile, Assistenzarzt, PPD Stadt Zürich

Dr. Maya Bose, Oberärztin, Spital Zimmerberg

Dr. Basil Caduff, Chefarzt, Spital Limmattal

Prof. Dr. Michele Genoni, Chefarzt,

Stadtspital Triemli

Dr. Beat Helbling, Leitender Arzt, Stadtspital Waid

Dr. Thomas H. Hess, Chefarzt,

Kantonsspital Winterthur

PD Dr. Emanuela Keller, Leitende Ärztin, UniversitätsSpital Zürich

Prof. Dr. Urs Lütolf, Ärztlicher Direktor,

UniversitätsSpital Zürich

Dr. Georg Mang, Chefarzt, Spital Bülach

Dr. Brigitte Muff, Chefärztin, Spital Bülach

PD Dr. Franco Salomon, Zürich

Dr. Adrian Schibli, Oberarzt, Stadtspital Triemli

Dr. Katharina Schiessl, Oberärztin,

UniversitätsSpital Zürich

Dr. Annelies Schnider, Leitende Ärztin,

Stadtspital Triemli

PD Dr. Stefan Wildi, Chefarzt, Stadtspital Waid

Mitverfasser des Verhaltenskodexes - unter Mitwirkung von Dr. Rudolf M. Reck, Zürcher Spitalärzte und Spitalärztinnen VSAO, und Dr. Alphons Beat Schnyder, MetaCultura 


\title{
Verhaltenskodex des Zürcher Kreises für fortschrittliches Spitalmanagement
}

\author{
Ärztliche Grundhaltung \\ - Wir nehmen unsere ärztliche und unternehmerische Verantwortung im Dienste der Patienten \\ und des gesellschaftlichen Gemeinwohls wahr. \\ - Für uns steht das Patientenwohl vor Eigeninteressen. \\ - Verhalten und Kommunikation in unserem Beruf orientiert sich an folgenden Werten: Integrität, \\ Transparenz, Glaubwürdigkeit, Menschlichkeit.
}

Ärztliche Diagnose und Behandlung

- Wir stellen das menschlich Sinnvolle über das technisch Machbare und erbringen optimale statt maximale Diagnose und Behandlung.

- Wir leben eine vertrauensvolle transparente Beziehung mit unseren Patienten. Wir holen die Meinung der Patienten ein und lassen sie beim Behandlungsprozess mitbestimmen.

- Ethisch schwierige Entscheidungen treffen wir nach sorgfältigem Abwägen, im Dialog mit Patienten und Angehörigen und nach bestem Wissen und Gewissen (in Anlehnung an das Modell «7 Schritte Dialog» der Stiftung Dialog Ethik).

Medizinische Qualität

- Unser Ziel ist die Sicherstellung einer guten medizinischen Versorgung der Bevölkerung und eine qualitativ hochstehende Behandlung der Patienten.

- Wo es sinnvoll und möglich ist, lassen wir unsere Qualität messen / wissenschaftlich überprüfen und legen Rechenschaft darüber ab.

- Wir sind proaktiv in der Definition und Erfassung von Qualitätsindikatoren.

Lehre, Förderung des medizinischen Nachwuchses

- Unser Fachwissen geben wir mittels Bildung und Förderung an den Nachwuchs weiter.

- Wir gestalten die ärztliche Weiterbildung effizient und effektiv. Unsere Weiterbildungsplätze sind attraktiv und wettbewerbsfähig.

- Wir setzen uns für eine faire Abgeltung von Leistungen für die ärztliche Aus-, Weiter- und Fortbildung ein.

Führungs- und Zusammenarbeitskultur im Spital

- Wertschätzung und Teamorientierung bilden das Fundament unserer Führungs- und Zusammenarbeitskultur.

- Unsere Entscheidungsprozesse sind transparent. Kaderärzte haben eine angemessene Mitsprache auf der jeweiligen Führungsebene.

- Wir setzen uns dafür ein, dass Aufgaben, Verantwortung und Kompetenzen kongruent, Leistung und Entlöhnung stimmig sowie Aufträge und Ressourcen im Einklang sind.

Ressourcen, Organisation, Innovation

- Wir sind ressourcenorientiert und wenden die Medizin mit dem besten Aufwand-Nutzen-Verhältnis an.

- Bei uns kommen neue medizinische Methoden/Therapien zum Einsatz, falls sie zu einem besseren oder zu einem gleichwertigen, aber kostengünstigeren Outcome führen.

- Wir rationalisieren statt rationieren und fördern den Einsatz von innovativen Techniken, Methoden und Prozessen mit kostensenkender Wirkung.

Ökonomie, Wettbewerb

- Wir stellen uns dem Wettbewerb. Wir verhalten uns fair gegenüber anderen Leistungsanbietern und Konkurrenten (betriebsintern und -extern).

- Leistungen werden nur erbracht, soweit sie medizinisch notwendig sind. Sie werden korrekt erfasst und abgerechnet. Wir bereichern uns nicht auf Kosten der Allgemeinheit.

- Wir wehren uns gegen eine Kommerzialisierung der Medizin und gehen vernünftig mit der Ökonomisierung der Medizin um.

Gesundheitspolitik, Reformprozess im Gesundheitswesen

- Wir setzen auf ein Miteinander statt Gegeneinander, erarbeiten konstruktive Lösungen und praktizieren partnerschaftliche Zusammenarbeit, Vernetzung und Kooperation.

- Wir unterstützen Reformen des Gesundheitswesens im Sinne unseres Ethos, setzen auf einen vertrauensvollen Umgang und wehren uns gegen ineffiziente Kontrollsysteme.

- Wir verfügen über Gelassenheit im Reformprozess und leisten unseren Beitrag zur Berechenbarkeit und Verlässlichkeit des Systems. 